\title{
Going Massive in education: Lessons learned from the first MOOC in Implant Dentistry
}

Nikos Mattheos ${ }^{1}$, Lan Min², Hou Xiangyu ${ }^{3}$, Qi Xinyu ${ }^{4}$, Seto Ellen ${ }^{4}$, Kwok Ricky ${ }^{3}$

1. Faculty of Dentistry, 2. Faculty of Education, 3. Faculty of Engineering, 4. TeLi, The University of Hong Kong

\section{What is a MOOC?}

\section{Why Implant Dentistry?}

\section{Massive Open Online Course -> MOOC}

Massive: thousands of participants with diverse backgrounds

Open: fully accessible for free to everyone

Online: fully online learning format

Course: thousands of courses created by elite universities

MOOCs are the latest developments in higher education,

supported by some of the leading global institutions through

large online platforms such as edX and Coursera.

The teaching of Implant Dentistry in the undergraduate curricula is limited, varies significantly between regions and institutions and many universities still have many difficulties to adequately implement it ${ }^{1}$. As a result, the majority of dental graduates in Asia and worldwide start their careers with a very limited understanding of dental implants as part of comprehensive care. Even long practicing dentists have little opportunity to reach flexible, quality-assured and unbiased education in this discipline ${ }^{2}$.

\section{Aims:}

$\checkmark$ Evaluate student demographics and learning behaviour in the first run of the MOOC G Investigate patterns of Self-Regulated Learning (SRL) behaviours through data mining $\checkmark$ Correlate SRL behaviours to learning performance and student characteristics

\section{Methods:}

$\square$ Big data from the first run of the MOOC (Oct 2016) of students' interactions and activities (clickstream) in the MOOC were quantitatively analysed.

G Zimmerman's theory ${ }^{3}$ was used to classify student engagement in a three phases: model: - forethought phase (i.e. preparing before study, introductory activities),

- performance phase (i.e. participating in activity, interaction with content, active learning), - self-reflection phase (i.e. evaluating/discussing performance).

Student's learning activity was modelled into these three phases based on the features of each of the learning activities. Effective SLR implied evidence of student attendance of activities in all 3 phases.

$\checkmark \mathrm{N}$-gram algorithm was applied to trace the frequency of SRL behaviours and the frequency of SRL loops. K-mean Cluster was applied to differentiate the more effective SRL learners and less effective SRL learners based on the frequency of SRL loops. T-test was used to check if the two effective level of SRL learners were significantly different.

\section{Results:}

In the first run of the course (25.10-5.12 2016):

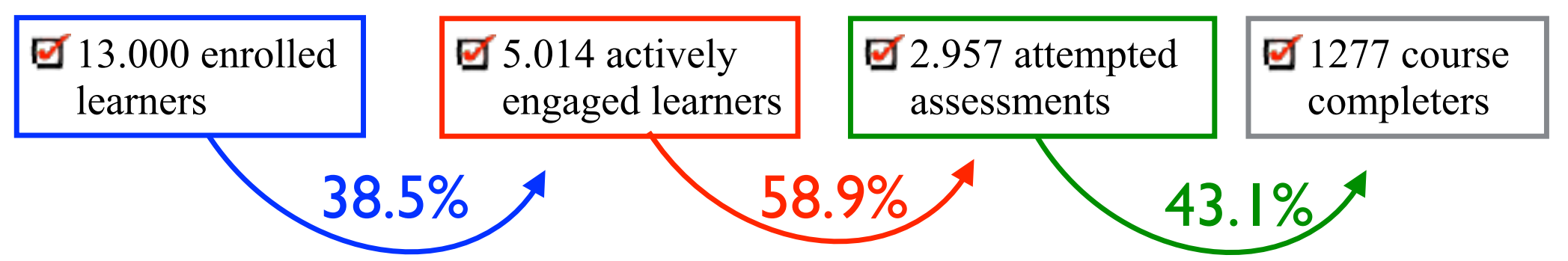

The majority of the learners demonstrated a "linear" learning behavior, following the course week by week. Half of them presented evidence of effective SLR (fig 1).

Among the linear learners, those with effective SLR achieved significantly higher grades $(0.64$ vs $0.58, \mathrm{p}<0.01)$. The same was not observed in the group of non-linear learners.

\section{References:}

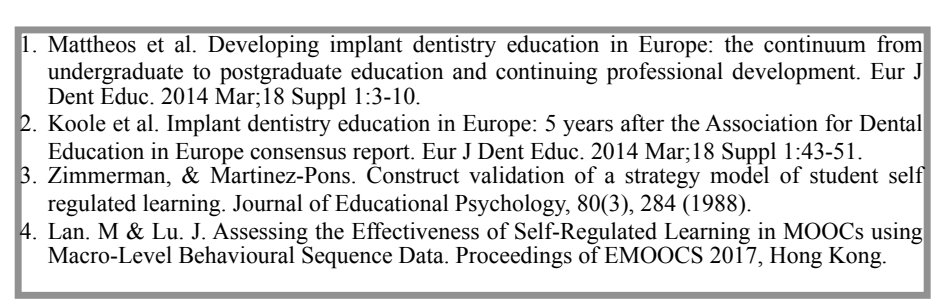

\section{Results:}

๑ A MOOC in Implant Dentistry attracted significant interest from learners

口 Most learners presented a "linear" learning pattern.

๑ Self Regulated Learning behaviour correlated with higher grades in linear learners, but not in the non-linear.
The HKU MOOC in Implant Dentistry

As of Sept 2017: 2 completed runs (10.2016, 2.2017) 23.319 enrolled learners 9758 active learners 1.903 completers $\quad 1.162$ certificate holders

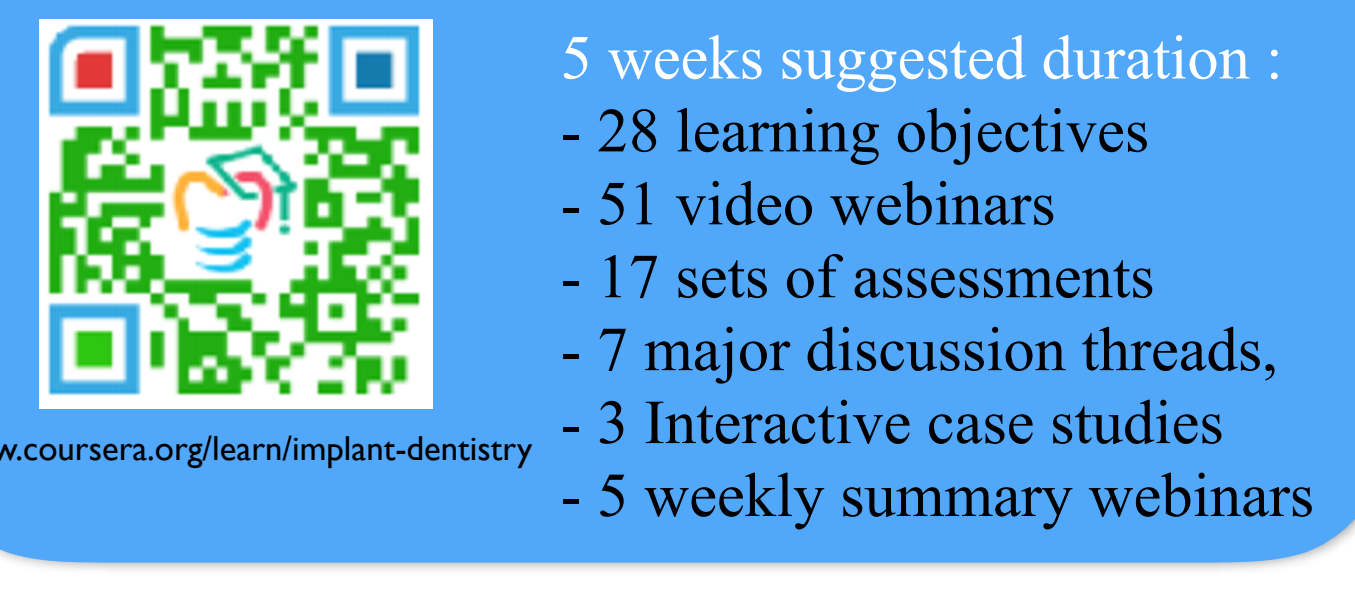

\section{Self-Regulated Learning}

Self-regulated learning is the degree to which students are metacognitively, motivationally, and behaviourally actively steering their own learning ${ }^{3}$. With less direct (face-to-face) help from instructors, online learners make decisions on what to learn and how to learn based on their self-regulation (e.g. interests, motivation). A MOOCs help instructors record all learning behaviours based on activities sequences, which is helpful to identify the ineffective SRL behaviours ${ }^{4}$.

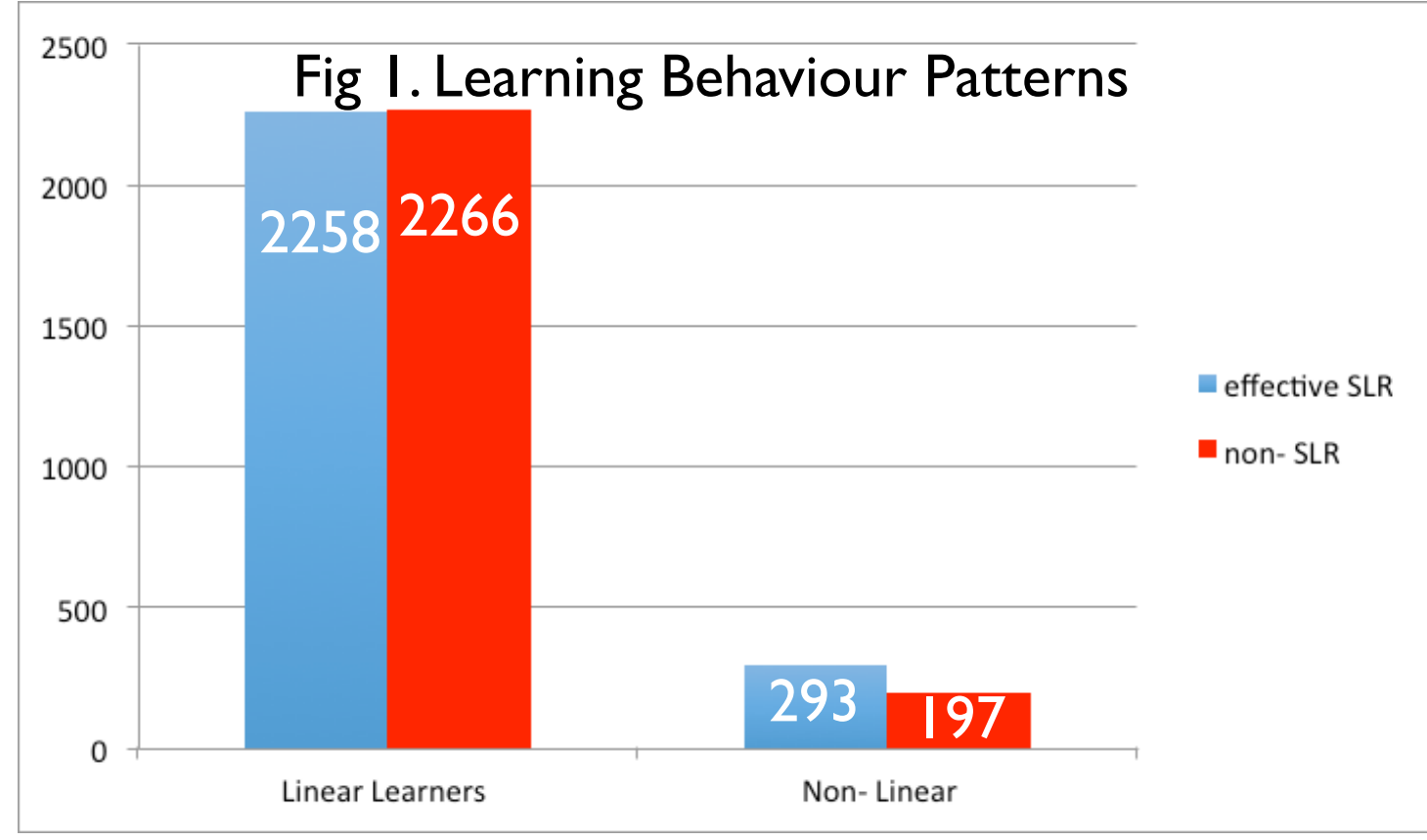

